\section{Hormonwirkung im Mann}

\section{Macht Soja impotent?}

Frage: Auf springermedizin.de wird berichtet, dass Soja in der Lage sei, Beschwerden von Patientinnen in der Peri-, bzw. Postmenopause signifikant zu senken. Es ist sicherlich davon auszugehen, dass diese Hilfe auf eine Hormonwirkung (Östrogen) von Soja zurück zu führen ist. Soja ist zunehmend in Gebrauch, auch bei Kindern und Männern, wo natürlich auch eine Hormonwirkung zu erwarten ist. Aus dieser Überlegung heraus, müsste man vor dem Verzehr von Soja und Produkten aus Soja (Tofu) nachdrücklich warnen. Kinder könnten bleibende Schäden in ihrer Entwicklung erleiden und bei Männern wäre wohl mit Gynäkomastie, Fettleber, Impotenz etc. zu rechnen. Oder?

Dr. Peter Stiefelhagen, Hachenburg: Die Tatsache, dass Soja klimakterische Beschwerden günstig beeinflusst, heißt ja noch lange nicht, dass es das Wirkprofil eines Östrogens hat, also bei Kindern und Männern unerwünschte Nebenwirkungen hervorrufen kann. Solche sind auch bisher nie beschrieben worden. Man sollte die Menschen also nicht mit solchen Überlegungen verunsichern.

Prof. Dr. Hermann S. FüeßI, Haar: In Populationen und Gegenden, wo der Genuss von Soja seit Jahrtausenden verbreitet ist, wie China oder Japan, wurden keine höheren Raten von Gynäkomastie, Impotenz, Fettleber beobachtet als anderswo auch. Allein dieser Umstand sollte doch überzeugen. Umgekehrt ist der Verzehr von großen Mengen tierischer Proteine, wie er für die westlichen Wohlstandsgesellschaften typisch ist, mit einer Reihe von Erkrankungen assoziiert wie Kolonkarzinom, Hypertonie, Übergewicht und Gicht. Lassen Sie sich nicht verrückt machen.

Expertenrat "MMW-Sprechstunde",

www.springermedizin.de

Jodhaltige Waterkant

\title{
Darf der Basedow-Patient ans Meer?
}

Frage: Wie sind Ihre Ernährungsempfehlungen bei manifester Hyperthyreose Basedow, sollte wegen der Jodbelastung ein Aufenthalt am Meer gemieden werden? Wie sind Ihre Empfehlungen bezüglich der Osteoporosevermeidung bei Basedow?

Prof. Dr. Johannes Köbberling, Wuppertal: Man darf die Jodmengen bei der Nahrungsaufnahme, in Deutschland durchschnittlich ca. 100-150 ug, in Amerika ca. 750 ug, nicht mit den sehr viel höheren Jodmengen, etwa durch Kontrastmittel, verwechseln, vor denen im Zusammenhang mit Schilddrüsenerkrankungen gewarnt wird. Ein Aufenthalt am Meer erhöht die "natürliche“ Jodaufnahme nur unwesentlich. Nichts spricht dagegen, dass Patienten mit Base- dow sich am Meer aufhalten. Es gibt auch keine diätetischen Einschränkungen. Das Wichtigste bei der Hyperthyreose ist eine schnelle Wiederherstellung des euthyreoten Zustandes, aber auch in der Phase bis zu diesem Zeitpunkt sind keine Einschränkungen erforderlich. (Anmerkung: es ist ohnehin fraglich, ob bei einem Basedow die Jodaufnahme eine Rolle spielt). Bezüglich Osteoporoseprophylaxe gilt alles das, was sonst gilt (und woran man glaubt). Nur eine längere Zeit bestehende Hyperthyreose würde ein gewisses zusätzliches Risiko darstellen, das muss aber ohnehin vermieden werden. Der behandelte Basedow ist kein Risiko.

Expertenrat Endokrinologie,

www.springermedizin.de

In vitro nachgewiesen

\section{Bakterizide und Bakteriostatika in Kombi?}

Frage: Wann und wann nicht und warum bestehen Kontraindikationen der Kombinationstherapie bei Antibiotika? Z.B. Makrolide und Cephalosporine bei CAP?

Dr. Anke Rolfes-Bußmann, Osnabrück, Prof. Dr. Jürgen C. Frölich, Hannover: Bakterizide Antibiotika wirken in der Regel am besten, wenn sie auf Bakterien zur Zeit der Teilung einwirken. Bei Kombination dieser Antibiotika mit bakteriostatisch wirkenden Antibiotika können letztere durch das langsamere Bakterienwachstum die bakterizide Wirkung schwächen. Dieses wurde überwiegend in in-vitro-Experimenten nachgewiesen. Grundsätzlich sollten diese Antibiotika nicht zusammen appliziert werden. In der Praxis haben sich jedoch verschiedene Kombinationen be- währt, die dieser allgemeinen Regel widersprechen: Bei schwerer ambulant erworbener Pneumonie wird eine Kombination von Makroliden (bakteriostatisch, Wirkung gegen atypische Erreger) und einem Cephalosporin der Gruppe 2/3 (bakterizid, Wirkung gegen Pneumokokken und Haemophilus influenzae) angewendet, um das Wirkungsspektrum zu erweitern (Beispiele: Erythromycin, Azithromycin oder Clarithromycin plus Cefuroxim, Cefotaxim oder Ceftriaxon). Auch bei der Helicobacter-pylori-Eradikation werden ein bakterizides (Amoxicillin) und ein bakteriostatisches Antibiotikum (Clarithromycin) kombiniert.

\footnotetext{
Expertenrat Pharmazie,
} www.springermedizin.de 